\title{
Editorial
}

J.E. Kasik, MD

\section{Skin Tests for Tuberculosis}

This issue of Infection Control contains the results of a survey of tuberculin skin test programs in long-term care facilities (LTCF) of a large geographic area, the state of North Carolina ${ }^{1}$ (pp 353-356). The report is both interesting and discouraging because a substantial proportion of the reporting hospitals have either inadequate programs or none at all. Although it remains to be established whether this occurrence is restricted to North Carolina, these findings may signal a widespread lack of compliance with established guidelines for tuberculosis (TB) control programs in LTCFs. This noncompliance may be caused by a lack of knowledge about the threat of tuberculosis in LTCFs, and education might motivate the administrators of these facilities to implement $\mathrm{TB}$ control programs. Alternately, it may be that these busy individuals do not perceive tuberculosis as a problem in their facility, at least not serious enough to warrant implementing what may seem to them a fairly complex program of tuberculin testing, recordkeeping, and follow-up. While data to support these possibilities are not available, I suspect that this report indicates a fairly widespread and profound apathy about the control of tuberculosis, based on the perception that tuberculosis is no longer a significant threat in LTCFs.

Certainly there has been a spectacular decline in the incidence of tuberculosis as a clinical problem in all developed countries in the last century. In many areas of the United States, tuberculosis has become almost a rare disease. ${ }^{2}$ The qualifier "almost" acknowledges the recent concerns related to tuberculosis occurring in special populations such as AIDS patients, intravenous drug users, and recent immigrants. ${ }^{3}$ Nevertheless, that reliable marker of the number of infectious cases of tuberculosis in a population, the prevalence of skin test reactivity in

From the Department of Internal Medicine, College of Medicine, University of Iowa, and the Veterans Administration Medical Center, Iowa City, Iowa.

The author acknowledges Ms. P. Foster for help with manuscript preparation.

Address reprint requests to J.E. Kasik, MD, IAO2, Veterans Administration Medical Center, lowa City, IA 52240. school children, had declined in the US until it was practically nonexistent; as a result, most of these programs have been discontinued. ${ }^{2}$

Despite this remarkable disappearance of what was formerly a much-feared and common illness, nearly everyone interested in tuberculosis control has remained concerned about the special problem of the nursing home population. ${ }^{4}$ Because of the average age of patients confined to LTCFs and the prevalence of other problems, such as mental illness and alcoholism, in this population, it has been widely believed that LTCFs remained at significant risk for outbreaks of tuberculosis. Because it is recognized that older individuals have lived during a time when tuberculosis was a common health problem, they should, and do, have a higher incidence of skin test reactivity to PPD (purified protein derivative) than younger populations. ${ }^{2.5}$ Alcoholics or patients who have been confined at some time to a mental hospital also have a relatively high risk of infection with Mycobacterium tuberculosis; because of their multiple social problems and their inability to follow chemotherapeutic or prophylaxic programs, they frequently escape control programs.

Infection with mycobacteria, as indicated by the conversion of skin test reactivity to $P P D$, signaled that the positive reactor harbored live but dormant mycobacteria and as a result, are at risk to develop active disease at any time. Because the elderly also may be immunosuppressed due to their age, occurrence of other diseases such as malignancy, or administration of certain drugs, they represent a higher risk than the general population for developing active disease. ${ }^{5}$ The conventional wisdom is that unless these tuberculin-positive individuals are identified and monitored with adequate follow-up, they could develop active pulmonary tuberculosis at any time which in turn could go unrecognized and pose a serious hazard to both staff and other patients. Because tuberculosis is spread by an infected aerosol generated by cough, the number of individuals potentially infected by a single active case could be large, particularly if ventilation is poor.

These facts should provide sufficient motivation for any 
administrator of an LTCF who wants to be vigilant about tuberculosis and should provide him or her with enthusiasm for a well-conceived and smoothly operating tuberculosis control program. The panic and news media attention that can arise as a result of an outbreak of tuberculosis in an LTCF is often very impressive and should provide additional incentive for administrators to establish TB control programs. Despite the potential risks, based on the data presented by Price and Rutala, ${ }^{1}$ some administrators of LTCFs either are not aware of the theoretical dangers of tuberculosis and the widespread concerns about this illness in LTCFs or these concerns have not been confirmed by their experience.

The latter, lack of reinforcement in the absence of outbreaks of active tuberculosis, cannot be established by the data provided by Price and Rutala. ' Certainly if there had been no significant outbreaks of tuberculosis in LTCFs in North Carolina for the last five years, it might be argued that elaborate programs for tuberculosis control might be superfluous and the effort involved better diverted to some other high priority areas. In part, this thesis may be supported by some of the data in their paper. On admission only $6.4 \%$ of the patients reported in the study had a positive test and the conversion rate for individuals admitted to LTCFs was only about $0.9 \%$ per year. A conversion rate of less than $1 \%$ is certainly not an epidemic if considered overall. However, they found a $16 \%$ conversion rate in one year in one facility. This fact should impress even the most skeptical person that problems exist. However, this number, a $16 \%$ conversion rate, must be used with some caution. Many facilities did not have programs that eliminate the problem of false-positive conversion of the PPD skin tests caused by the booster effect of the test itself.

For those who are not familiar with the PPD-related booster phenomenon or booster effect, it is a condition found in individuals who at some time have been infected with $M$ tuberculosis and have developed a positive skin test. Over time their immunity, in absence of sufficient new antigenic stimulation, fades. When the individual is later tested with PPD, the test is negative; however, the antigen in the PPD stimulates sufficient immunological recall so that if the person is tested again, for example, six months later, he or she will now have a positive test. These results could lead to that person being considered a converter, infected with mycobacterium since the first test, triggering case-finding efforts and perhaps leading to inappropriate prophylaxis for that person.

It has been suggested by several groups $6-8$ that this problem of false-positive tests produced by the booster effect could be minimized by an initial evaluation of two tests spaced one week apart. Those who are positive PPD reactors on the second test should not be considered true conversions and not recently infected, because a week or two is too brief a period to allow infection and conversion in a previously uninfected individual.

This approach has never been popular and most of the hospitals reported in the study did not follow this recommendation. As a result, the $16 \%$ conversion rate noted may be lower since an incidence of booster phenomenon has been reported to be about $5 \%$ in LTCF patients. ${ }^{4}$ If one discounts that percent of apparent positives as false, the residual annual conversion rate would be about $10 \%$. This conversion rate would still be very impressive and would, in my opinion, signal a serious outbreak and should initiate case-finding efforts, modification of sanitation procedures, and perhaps prophylaxis with drugs. The prevention of spread of disease, as well as prompt. identification of patients who might die from an easily treated illness, are the major goals of any tuberculosis control program.

Of course, these are the critical points. Does a fully implemented tuberculosis control program, including at comprehensive skin test program for both patients and employees, prevent outbreaks of tuberculosis? Certainly this is the current and widely held wisdom, but real data to support the proposition that a well-developed program will deliver relative freedom from problems is absent and neglects a salient point. When a skin test program has identified a positive reactor and if this individual is properly evaluated with a chest $x$-ray, it should eliminate unknowing admission of active cases. It would not, however, prevent admission of contagious patients who are immunosuppressed. While someone with, for example, AIDS should be easy to recognize, other illnesses may not be as apparent. Immunosuppressive drugs or widespread carcinoma may be just as effective as AIDS in suppressing skin test reactivity. Individuals with these problems also can develop active disease if they harbor $M$ uberculosis. Unless the physician evaluating the patient remembers this, the initial evaluation will not be of value and, on the contrary, only provide an illusion of security.

Another defect in the current recommendations for tuberculosis concerns follow-up. The repeat of semiannual or annual skin testing in PPD-negative persons is beneficial because it should indicate an outbreak of tuberculosis and limit its spread when the source case is identified and isolated. It will not prevent outbreaks if the individuals who have a positive skin test in the facility are not recognized as a special risk and if this fact is not recalled should they become ill, particularly with an obscure illness among the background of other serious illnesses found in LTCF patients. Routine follow-up chest $\mathrm{x}$-rays, even if obtained twice a year, leave long intervals when cough, low-grade fever, weight loss, or other symptoms that should trigger reevaluation, can be ignored. A positive reaction to PPD, which should lead to the identification of patients at risk, is useful; but the information must be applied with the goal of preventing the spread of tuberculosis in a confined environment.

Because of these apparent difficulties and the low incidence of active tuberculosis, there has been discussion that the recommendations for LTCFs be modified and simplified. Recently, the state of Iowa changed the regulations for tuberculosis control in L.TCFs. The new rules discard the older, comprehensive approach, especially follow-up testing. Persons admitted to an LTCF must be evaluated for tuberculosis on admission although followup skin testing is not now required. Apparently "evaluation" means either skin testing or a recent chest $x$-ray. Because patients admitted to LTCFs usually come from hospitals, where chest $x$-rays are fairly routine, skin testing may not be common and certainly is not universal. As 
a result, a patient infected with mycobacteria that has inactive disease unapparent on $x$-ray could be admitted and not recognized as a special risk.

In defense of the changes made in Iowa, there has been no known outbreak of tuberculosis in an LTCF in Iowa for over five years (personal communication, C. Christie, RN, Iowa Department of Public Health, 1987). During much of that period, however, there were requirements for a comprehensive testing program, which were followed, more or less, in some facilities of this state. It should also be pointed out that Iowa has one of the lowest incidences of tuberculosis in this country, and that the state is not beset with some of the health and social problems often found in larger cities. As a result, Iowa may be a poor model for other areas.

What about the rest of the country? Based on the report by Price and Rutala in this issue and on other reports, $9-11$ it would seem that recommending cessation of routine skin testing programs for comprehensive tuberculosis control in LTCFs would be both premature and reckless. Only when there are data to show that routine comprehensive programs in LTCFs, including testing on admission and follow-up, are not worth the effort and do not prevent outbreaks of tuberculosis in LTCFs, should a firm recommendation be made. In places with many recent immigrants or with other significant social factors, I would recommend vigorous continuation of comprehensive programs until it can be demonstrated in that special environment whether or not it is worth the effort.

Finally, there are some parts of this country where a comprehensive tuberculosis control program in LTCFs should continue regardless of any recommendations. The effect of a high prevalence of AIDS on the control of tuberculosis may create a high-risk situation, the impact of which is still unknown. Considering the effects of a combination of immunosuppression, prevalence of abnormal chest $\mathrm{x}$-rays in patients with AIDS, lack of classical symptoms of active tuberculosis found in immunosuppressed individuals who could go unrecognized, there is an explosive potential for infecting many people. While skin testing would not prevent some of the more frightening aspects of this problem from developing, it would at least provide data as to the impact on health care personnel and other patients and allow further control measures to be developed.

There is an old Chinese curse, "May you live in interesting times."

\section{REFERENCES}

1. Price LE, Rutala WA: Tuberculosis screening in the long-term care setting. Infect Control 1987; 8:353-356.

2. Stead WW, Dutt AK: Epidemiology and host factors, in Schlossberg D (ed): Tuberculosis. New York, Praeger Monographs in Infectious Diseases, 1983, p 3.

3. Centers for Disease Control, US Dept of Health and Human Services: Diagnosis and management of mycobacterial infection and disease in persons with human immumodeficiency virus infection. Ann Intern Med 1987: 106:254-256.

4. Stead WW, Lofgren JP, Warren E: Tuberculosis as an endemic and nosocomial infection among elderly in nursing homes. $N$ Engl J Med 1985: 312:1483-1487.

5. Tuberculosis in old age, editorial. Tubercle $1983 ; 64: 69-71$.

6. Thompson NJ, Glassworth JL, Snider DL, et al: The booster phenomenon in tuberculin skin testing. Am Rev Respir Dis 1979; 119:587-597.

7. McGowan JE: The booster effect: A problem for surveillance of uberculosis in hospital employees. Infect Control 1980; 1:147-149.

8. American Thoracic Society: The tuberculin skin test. Am Rev Respir Dis 1981; 124:356-363.

9. Stead WW: Tuberculosis among the elderly: An outbreak in a nursing home. Ann Intern Med 1981; 94:606-610.

10. Centers for Disease Control: Tuberculosis in nursing care facility: $M M W R$ $1983 ; 32: 121$.

11. Anderson HR: Tuberculosis in nursing homes. I Tenn Med Assoc 1985; 70:765-766. 